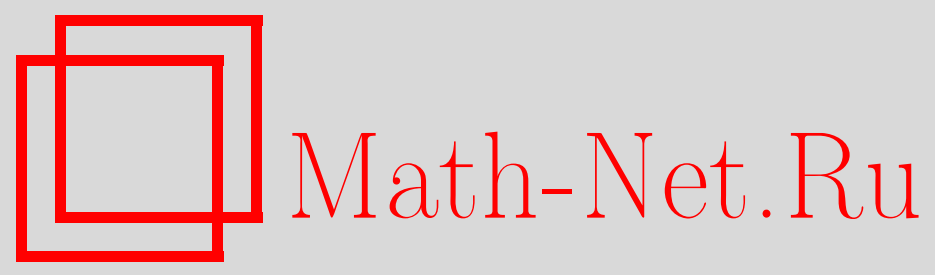

И. В. Коннов, Игра ограничений для оценки гарантированной работоспособности сложной системы, Изв. вузов. Матем., 2019, номер 4, 89-94

DOI: https://doi.org/10.26907/0021-3446-2019-4-89-94

Использование Общероссийского математического портала Math-Net.Ru подразумевает, что вы прочитали и согласны с пользовательским соглашением

http://www . mathnet.ru/rus/agreement

Параметры загрузки:

IP : 52.205 .19 .152

26 апреля 2023 г., 15:48:30 
Известия вузов. Математика

2019, № 4, с. 89-94

Краткое сообщение http://kpfu.ru/science/nauchnye-izdaniya/ivrm/

e-mail: izvuz.matem@kpfu.ru

\title{
И.В. КОННОВ
}

\section{ИГРА ОГРАНИЧЕНИЙ ДЛЯ ОЦЕНКИ ГАРАНТИРОВАННОЙ РАБОТОСПОСОБНОСТИ СЛОЖНОЙ СИСТЕМЫ}

\begin{abstract}
Аннотация. Предлагается постановка игровой задачи для оценки гарантированной работоспособности составной системы в условиях возможных внешних воздействий и при наличии ресурсов защиты. Для полученной задачи предлагается комбинация методов штрафов и негладкой оптимизации, позволяющая существенно упростить процесс решения по сравнению с поиском гарантированной стратегии в обычной игре.

Ключевые слова: игра ограничений, оценка работоспособности системы, антагонистические игры, негладкая оптимизация, метод штрафов.
\end{abstract}

УДК: 519.85

DOI: $10.26907 / 0021-3446-2019-4-89-94$

\section{ВВеДЕНИЕ}

Вначале рассмотрим общую задачу оценки производительности некоторой системы, содержащей $m$ подсистем. В этой системе могут выполняться (обрабатываться) $n$ типов заданий (продуктов) различного объема, причем каждый тип заданий выполняется своим набором подсистем (или несколькими возможными наборами) с определенным порядком и уровнем загрузки. Для заданного профиля объемов $x=\left(x_{1}, \ldots, x_{n}\right)^{\top}$ значение функции $h_{i}(x)$ определяет превышение мощности $i$-й подсистемы, $i=1, \ldots, m$. Если $X$ определяет множество всех профилей объемов, то можно определить их допустимое множество

$$
D=\left\{x \in X \mid h_{i}(x) \leq 0, i=1, \ldots, m\right\} .
$$

Пусть значение функции $\mu(x)$ определяет величину полезной работы системы для профиля объемов $x$. Тогда оценка производительности системы может быть найдена в результате решения задачи оптимизации

$$
\max _{x \in D} \rightarrow \mu(x) .
$$

Теперь предположим, что мощность подсистем может меняться под влиянием воздействий как для ограничения их работы, так и для защиты от такого вмешательства. Пусть имеются $t$ областей системы, на которых может оказываться воздействие для ограничения работы подсистем, а также $s$ областей системы, где может оказываться защитное воздействие, т. е. эти области не обязательно совпадают друг с другом и с подсистемами. Пусть

Поступила в редакцию 21.10.2018, после доработки 21.10.2018. Принята к публикации 19.12.2018

Благодарности. Работа выполнена в рамках выполнения государственного задания Минобрнауки России, номер задания 1.460.2016/1.4. 
$V \in \mathbb{R}^{t}$ определяет множество всех профилей объемов воздействия для ограничения мощностей, $U \in \mathbb{R}^{s}$ определяет множество всех профилей объемов защиты. Для заданной пары $(u, v) \in U \times V$ и профиля объемов $x$ превышение мощности $i$-й подсистемы будет теперь определяться значением функции $h_{i}(u, v, x)$, т. е. аналогично (1) оценка производительности системы определяется с помощью задачи оптимизации

$$
\max _{x \in D(u, v)} \rightarrow \mu(x),
$$

где $D(u, v)=\left\{x \in X \mid h_{i}(u, v, x) \leq 0, i=1, \ldots, m\right\}$. Оптимальное значение целевой функции в (2) можно обозначить $H(u, v)$ и определить антагонистическую игру с функцией выигрыша $H$ и множествами стратегий игроков $U$ и $V$.

Отметим, что в большинстве работ, связанных с играми типа «нападение-защита», предполагается известной формула для вычисления значения функции выигрыша для любых пар стратегий (например, [1]-[4]). Это позволяет достаточно просто находить решение игры, несмотря на присутствие обычно только одного из свойств вогнутости-выпуклости у функции $H(u, v)$. Здесь же решение игры сильно затруднено тем, что для вычисления значения функции выигрыша требуется решать достаточно сложную задачу оптимизации (2), поскольку реальные системы могут содержать большое число блоков (подсистем) со сложной структурой взаимосвязей. Один из подходов к решению в этих условиях состоит в предположении, что число стратегий игроков конечно и невелико, что сокращает объем вычислений. Например, в ([4], §2.13) такой подход (для второго игрока) применен при базовой задаче о максимальном потоке через сеть.

В настоящей работе предлагается модифицировать постановку игровой задачи, что позволит оценивать гарантированную работоспособность системы в общем случае, а также построить довольно простые методы поиска решения.

\section{1. ПОСТАНОВКА ЗАДАЧИ И МЕТОД ШТРАФОВ}

Будем использовать следующие предположения.

(A1) $X, U$ и $V$ - непустые компактные множества в $\mathbb{R}^{n}, \mathbb{R}^{s}$ и $\mathbb{R}^{t}$.

(A2) $\mu: X \rightarrow \mathbb{R}$ и $h_{i}: U \times V \times X \rightarrow \mathbb{R}, i=1, \ldots, m,-$ непрерывные функции.

Определим функцию штрафа $P(u, x)=\max _{v \in V} \max \left\{h_{1}(u, v, x), \ldots, h_{m}(u, v, x), 0\right\}$ и задачу оптимизации

$$
\max _{(u, x) \in W} \rightarrow \mu(x)
$$

где $W=\left\{(u, x) \in U \times X \mid P(u, x)=P^{*}\right\}, P^{*}=\min _{(u, x) \in U \times X} P(u, x)$. Отметим, что при наличии пары $(u, x) \in U \times X$, для которой выполняются ограничения по мощности всех блоков при любом выборе $v \in V$, получаем $P^{*}=0$. Тогда задача состоит в определении профилей объемов защиты и объемов заданий, обеспечивающих наибольшую гарантированную производительность системы независимо от профилей ограничений. При отсутствии такой пары выявляются «узкие места» структуры системы, защиту которых следует повысить.

Обозначим

$$
f(x)=-\mu(x), \Psi(u, x, \tau)=f(x)+\tau P(u, x),
$$

а также

$$
\mu^{*}=\max _{(u, x) \in W} \mu(x)
$$


Метод штрафов. Выбираем пару $\left(u^{0}, x^{0}\right) \in U \times X$, последовательности чисел $\left\{\varepsilon_{k}\right\} \searrow 0$, $\left\{\tau_{k}\right\} \nearrow+\infty$. На $k$-й итерации, $k=1,2, \ldots$, определяем пару $\left(u^{k}, x^{k}\right)$ такую, что

$$
\left(u^{k}, x^{k}\right) \in U \times X, \Psi\left(u^{k}, x^{k}, \tau_{k}\right) \leq \Psi_{k}^{*}+\varepsilon_{k},
$$

где $\Psi_{k}^{*}=\min \left\{\Psi\left(u, x, \tau_{k}\right) \mid(u, x) \in U \times X\right\}$.

Теорема. При условиях $(A 1)-(A 2)$ последовательность пар $\left\{\left(u^{k}, x^{k}\right)\right\}$ имеет предельные точки, и все они являются решениями задачи (3), кроме того,

$$
\lim _{k \rightarrow \infty} \mu\left(x^{k}\right)=\mu^{*} .
$$

В силу компактности множеств $U$ и $X$ последовательность $\left\{\left(u^{k}, x^{k}\right)\right\}$ ограничена, поэтому она имеет предельные точки. Для любой пары $\left(u^{*}, x^{*}\right) \in W$ согласно (5) имеем

$$
f\left(x^{k}\right)+\tau_{k} P\left(u^{k}, x^{k}\right) \leq \Psi_{k}^{*}+\varepsilon_{k} \leq f\left(x^{*}\right)+\tau_{k} P\left(u^{*}, x^{*}\right)+\varepsilon_{k} \leq f\left(x^{*}\right)+\tau_{k} P\left(u^{k}, x^{k}\right)+\varepsilon_{k},
$$

отсюда следует $0 \leq P\left(u^{k}, x^{k}\right) \leq P\left(u^{*}, x^{*}\right)+\left[f\left(x^{*}\right)-f\left(x^{k}\right)+\varepsilon_{k}\right] / \tau_{k} \rightarrow P^{*}$ при $k \rightarrow \infty$. Если $(\bar{u}, \bar{x})$ - любая пара предельных точек для $\left\{\left(u^{k}, x^{k}\right)\right\}$, то $(\bar{u}, \bar{x}) \in W$. Далее, из $(7)$ также имеем $f\left(x^{k}\right) \leq f\left(x^{*}\right)+\varepsilon_{k}$, поэтому $\mu(\bar{x})=\mu^{*}$. Отсюда следует (6).

Таким образом, задача (3) сводится к приближенному решению последовательности задач вида

$$
\min _{(u, x) \in U \times X} \rightarrow \Psi(u, x, \tau) .
$$

При этом в случае $P^{*}=0$ получаем обычный метод штрафов. Тогда соотношение (6) можно усилить, поскольку из (7) также следует

$$
\lim _{k \rightarrow \infty} f\left(x^{k}\right)=\lim _{k \rightarrow \infty} \Psi\left(u^{k}, x^{k}, \tau_{k}\right)=\lim _{k \rightarrow \infty} \Psi_{k}^{*}=f^{*}=-\mu^{*} .
$$

Отметим, что применение методов штрафов (регуляризации) для задач последовательной оптимизации хорошо известно [5], [6]. При этом дополнительные условия типа регулярности позволяют достичь решения и при конечном значении параметра штрафа $\tau$.

\section{2. УСЛОВИЯ РЕАЛИЗАЦИИ МЕТОДА}

Теперь обсудим особенности реализации предложенного метода.

Предложение. Пусть выполняется условие $(A 1), \eta_{i}: V \rightarrow \mathbb{R}, i=1, \ldots, p,-$ непрерывные функции. Тогда

$$
\max _{v \in V} \max _{i=1, \ldots, p} \eta_{i}(v)=\max _{i=1, \ldots, p} \max _{v \in V} \eta_{i}(v) .
$$

Действительно,

$$
\exists \bar{v} \in V, \eta^{\prime}=\max _{v \in V} \max _{i=1, \ldots, p} \eta_{i}(v)=\max _{i=1, \ldots, p} \eta_{i}(\bar{v}) \leq \max _{i=1, \ldots, p} \max _{v \in V} \bar{\eta}_{i}=\eta^{\prime \prime},
$$

где $\bar{\eta}_{i}=\max _{v \in V} \eta_{i}(v)$. Но также $\eta^{\prime \prime}=\bar{\eta}_{l}=\eta_{l}(\tilde{v})$ для некоторых $l, \tilde{v} \in V$. Поскольку $\eta_{i}(\tilde{v}) \leq \bar{\eta}_{i} \leq$ $\bar{\eta}_{l}$ при $i \neq l$, то

что и требовалось.

$$
\eta^{\prime \prime}=\max _{i=1, \ldots, p} \eta_{i}(\tilde{v}) \leq \max _{v \in V} \max _{i=1, \ldots, p} \eta_{i}(v)=\eta^{\prime}
$$

Отметим, что подобное свойство при несколько иных условиях было получено в ([2], с.267). Из предложения 2 следует

$$
P(u, x)=\max \left\{\max _{v \in V} h_{1}(u, v, x), \ldots, \max _{v \in V} h_{m}(u, v, x), 0\right\} .
$$

Теперь уточним используемые предположения. 
(B1) $X, U$ и $V$ - непустые, выпуклые и компактные множества в $\mathbb{R}^{n}, \mathbb{R}^{s}$ и $\mathbb{R}^{t}$.

(B2) $\mu: X \rightarrow \mathbb{R}$ - непрерывная вогнутая функция, $h_{i}: U \times V \times X \rightarrow \mathbb{R}, i=1, \ldots, m$, непрерывные функции, выпуклые по переменным $u, x$ и вогнутые по переменной $v$.

Тогда выполняются условия (A1)-(A2), задачи (3) и (8) суть задачи выпуклой оптимизации. Кроме того, вычисление значения функции штрафа $P(u, x)$ согласно $(9)$ состоит в решении $m$ независимых задач выпуклой оптимизации вида

$$
\max _{v \in V} \rightarrow h_{i}(u, v, x) .
$$

Для решения задачи (8) можно применить подходящий метод выпуклой негладкой оптимизации (например, [7], [8]). Таким образом, при сделанных общих предположениях метод может быть достаточно просто реализован.

Если в условии (В2) вместо вогнутости потребовать выпуклость функций $h_{i}(u, v, x)$, $i=1, \ldots, m$, также по переменной $v$, то, как нетрудно показать, функция выигрыша $H(u, v)$ исходной антагонистической игры, которая определяется как оптимальное значение целевой функции в задаче (2), будет вогнутой по обеим переменным. Тогда первый игрок будет иметь чистую оптимальную стратегию, а второй - только смешанную с конечным спектром (например, [3], [4]). Но при этом вычисление оптимальной стратегии первого игрока с помощью задачи

$$
\max _{u \in U} \min _{v \in V} \rightarrow H(u, v)
$$

будет включать необходимость решения задачи невыпуклой (глобальной) минимизации по переменной $v$ для каждого значения $u$, помимо решения задачи (2). Ситуация не изменится, если функции $h_{i}(u, v, x), i=1, \ldots, m$, будут линейными по переменной $v$. Тогда выполняются условия (B1)-(В2). Отсюда можно заключить, что предлагаемая модификация постановки существенно упрощает поиск решения по сравнению с решением исходной игры.

Замечание. Предложенный подход может быть применен для оценки работоспособности и устойчивости к воздействиям систем различной природы, имеющих достаточно большое число подсистем со сложной структурой взаимосвязей. $\mathrm{K}$ ним можно отнести различные финансовые, транспортные, экономические и промышленные системы, для них можно проверять устойчивость к воздействиям, которые могут ограничить мощности их подсистем и осложнить их работу в целом, а также эффективность наличных защитных мер. Также можно использовать данную модель для проверки устойчивости при проектировании больших технических систем, имеющих сложную многосвязную структуру. Достаточно удобно применять предложенный подход при выработке мер защиты систем связи и компьютерных сетей, поскольку их сложная топология и большая размерность не позволяют, как правило, получать явные формулы для оценки каждой отдельной ситуации и давать аналитическое решение возникающих задач.

В качестве иллюстрации приведем приложение к задаче оптимизации потоков в компьютерных и телекоммуникационных сетях передачи данных на примере одной из основных моделей распределения потоков [9]. Для определенного периода времени, в сети, содержащей $m$ линий передачи (дуг), выполняются заявки на передачу потоков для выделенных $n$ пар вершин, при этом $x_{j}$ и $d_{j}$ - текущее и максимальное значения объема заявки $j, c_{i}$ - пропускная способность дуги $i$. Для упрощения предполагается, что для каждой заявки $j$ выделен единственный маршрут передачи данных, в который входят дуги с индексами из множества $M(j)$, поэтому для каждой дуги $i$ однозначно определяется множество пар 
вершин $N(i)$, маршруты передачи которых содержат эту дугу. Для каждой заявки $j$ объем передачи $x_{j}$ дает значение полезности $\sigma_{j}\left(x_{j}\right)$. Тогда задача максимизации суммарной полезности приобретает вид

$$
\max \rightarrow\left\{\sum_{j=1}^{n} \sigma_{j}\left(x_{j}\right) \mid \sum_{j \in N(i)} x_{j} \leq c_{i}, i=1, \ldots, m, 0 \leq x_{j} \leq d_{j}, j=1, \ldots, n\right\} .
$$

При вогнутых функциях $\sigma_{j}\left(x_{j}\right)$ получаем задачу выпуклой оптимизации на многограннике.

Предположим, что пропускная способность дуги $i$ может меняться под влиянием воздействий как на $t$ пунктов (вершин, дуг) сети для ограничения работы, так и со стороны $s$ пунктов сети, где может оказываться защитное воздействие, т. е. $c_{i}=c_{i}(u, v)$ при профилях защиты-вмешательства $(u, v) \in U \times V$. Множества $U, V$ профилей объемов воздействия обычно определяются как многогранники, например,

$$
\begin{aligned}
& U=\left\{u \in \mathbb{R}^{s} \mid 0 \leq u_{i} \leq \alpha_{i}, i=1, \ldots, s, \sum_{i=1}^{s} u_{i} \leq C^{\prime}\right\}, \\
& V=\left\{v \in \mathbb{R}^{t} \mid 0 \leq v_{j} \leq \beta_{j}, j=1, \ldots, t, \sum_{j=1}^{t} v_{j} \leq C^{\prime \prime}\right\} .
\end{aligned}
$$

Задача (8), (4) приобретает вид

$$
\min _{(u, x) \in U \times X} \rightarrow \Psi(u, x, \tau)=\left\{-\sum_{j=1}^{n} \sigma_{j}\left(x_{j}\right)+\tau P(u, x)\right\},
$$

где $X=\left\{x \in \mathbb{R}^{n} \mid 0 \leq x_{j} \leq d_{j}, j=1, \ldots, n\right\}$, а также согласно (9)

$$
P(u, x)=\max \left\{\sum_{j \in N(1)} x_{j}-\min _{v \in V} c_{1}(u, v), \ldots, \sum_{j \in N(m)} x_{j}-\min _{v \in V} c_{m}(u, v), 0\right\} .
$$

При вогнутых функциях $\sigma_{j}\left(x_{j}\right)$ и $c_{i}(\cdot, v)$ получаем задачу выпуклой негладкой оптимизации (10) на многограннике. При выпуклых функциях $c_{i}(u, \cdot)$ вычисление значения функции штрафа $P(u, x)$ согласно (11) будет по существу состоять в решении $m$ независимых задач выпуклой оптимизации вида

$$
\min _{v \in V} \rightarrow c_{i}(u, v)
$$

Кроме того, если переменные в этих функциях разделимы, например, когда

$$
c_{i}(u, v)=c_{i}^{\prime}+a_{i}(u)-b_{i}(v) \text { или } c_{i}(u, v)=a_{i}(u) / b_{i}(v), a_{i}(u)>0, b_{i}(v)>0,
$$

то будет достаточно решить каждую задачу выпуклой оптимизации (12) только один раз и использовать эти решения для всех значений $u, x$. Таким образом, задача оптимизации $(10)$ тогда упрощается и ее решение может быть найдено при достаточно большой размерности, а это позволяет найти стратегию максимальной гарантированной полезности сети связи.

\section{ЛитеРАтУРА}

[1] Дрешер М. Стратегические игры, Советское радио, М., 1964.

[2] Гермейер Ю.Б. Введение в теорию исследования операций, Наука, М., 1971.

[3] Воробьев Н.Н. Основъ теории игр. Бескоалиционнъе игри, Наука, М., 1984.

[4] Давыдов Э.Г. Исследование операций, Высш. шк., М., 1990.

[5] Еремин И.И. О задачах последователъного программирования, Сиб. матем. журн. 14:1 (1973), 53-63. 
[6] Федоров В.В. Численнъе методи максимина, Наука, М., 1979.

[7] Демьянов В.Ф., Васильев Л.В. Недифференцируемая оптимизация, Наука, М., 1981.

[8] Коннов И.В. Нелинейная оптимизация и вариационные неравенства. - Казань: Казанск. ун-т, 2013.

[9] Kelly F.P., Maulloo A., Tan D. Rate control for communication networks: shadow prices, proportional fairness and stability, J. Oper. Res. Soc. 49:3 (1998), 237-252.

\section{Игорь Васильевич Коннов}

Казанский федеральный университет,

ул. Кремлевская, д. 18, г. Казань, 420008, Россия,

e-mail: konn-igor@yandex.ru

\section{I.V.Konnov}

\section{Game of constraints for evaluation of guaranteed composite system performance}

Abstract. We propose a game problem formulation for evaluation of guaranteed composite system performance under possible external interference and in the presence of protection resources. The problem suggested to be solved by combined penalty and non-smooth optimization methods. This enables one to essentially simplify the solution process in comparison with finding worst case strategies in the custom zero-sum game.

Keywords: game of constraints, evaluation of system performance, zero-sum games, non-smooth optimization, penalty method.

Igor Vasilyevich Konnov

Kazan Federal University, 18 Kremlyovskaya str., Kazan, 420008 Russia,

e-mail : konn-igor@yandex.ru 